\title{
MODELLING FIRE SEVERITY AND EVACUATION IN TUNNELS
}

Adequate design methods and tools are vital to any construction project, especially when its complexity and value are high. Tunnels, road or railway, are a good example of such structures. This paper deals with the topic of computer modelling utilization in the design and evaluation of fire safety in tunnels. The capabilities of selected software - Fire dynamics simulator + Evac - are demonstrated on a practical example - Tunnel Lucivna in the Slovak Republic. In this example case, both fire and evacuation modelling is used in conjunction to assess the overall fire safety of the tunnel. The results show the effects of fire on the tunnel structure as well as the persons inside the tunnel trying to evacuate.

Keywords: Fire severity, computer fire modeling, evacuation, tunnel fires, smoke movement.

\section{Introduction}

This paper is the third one in a series of papers dealing with computer modelling of fires in tunnels. The first paper [1] is an introduction to the topic and deals with a simplified scenario to demonstrate computer modelling capabilities in this area. In the second paper [2] full detailed scenario is modelled and further parameters evaluated. Current work goes even further and incorporates evacuation modelling and evaluates the effect of the boundary conditions at the tunnel's portals.

Tunnel fires are very different from fires within 'standard' buildings due to their specific construction. This, in combination with potentially large fire loads, such as fully laden lorries, creates conditions for very severe fires of tens of megawatts. It is therefore necessary that every tunnel is designed to withstand a realistic worst case fire and allows for safe evacuation and effective firefighting.

Computer fire modelling is a very suitable tool for this purpose. After over three decades of application of computer based information systems to the crisis management, these systems are getting wider acceptance by the community of the emergency managers and designers [3].

\subsection{Fire as a major hazard and threat to tunnels}

It was stated previously that tunnels are quite vulnerable to fire, be it accidentally or deliberately started, which is primarily due to their specific construction and the ease of access.

A recent study entitled Making transportation tunnels safe and secure [4] evaluated the various hazards and threats that may adversely affect transportation tunnels. To give a concise overview of the hazards and threats, they are tabulated, together with their potential to damage the normal operation of a transportation system, including specific tunnel components (Table 1).

Major hazards and threats to transportation tunnels

and associated features [4]

Table 1

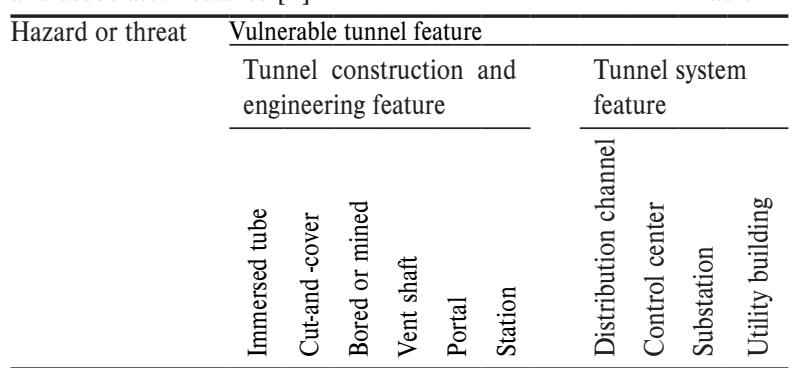

Hazard

\begin{tabular}{llllllllllll}
\hline Fire (unintentional) & $\checkmark$ & $\checkmark$ & $\checkmark$ & $\checkmark$ & $\checkmark$ & $\checkmark$ & $\checkmark$ & $\checkmark$ & $\checkmark$ & $\checkmark$
\end{tabular}

Structural integrity

loss by natural $\checkmark \quad \checkmark \quad \checkmark \quad \checkmark \quad \checkmark \quad \checkmark \quad r \quad \checkmark \checkmark \checkmark$

causes

Introduction of $\checkmark \checkmark \checkmark \checkmark \checkmark \checkmark$

hazardous materials

Threat

Small IED

Medium-sized IEDs

Large IEDs

Chemical agents

Biological agents

Radiological agents

Cyber attack

Maritime Incident

Fire (arson)

Sabotage of MEC

systems

IED - Improvised explosive device

MEC- mechanical, electrical and communication

\footnotetext{
* Vladimir Mozer

Department of Fire Engineering, Faculty of Special Engineering, University of Zilina, Slovakia, Email: vladimir.mozer@fsi.uniza.sk
} 
It is clear that fire has the most destructive potential, along with medium and large improvised explosive devices (which can lead to a fire too) The table lists the various types of tunnels, as well as their engineering and system features that may be affected. Thus, there is a possibility that a fire may take the tunnel out of service. The duration of the service disruption, of course, depends on the extent of damage caused. Nonetheless, it is not just the tunnel itself that is affected, but also the transportation network the tunnel forms a vital part of. This is particularly the case of tunnels forming part of critical infrastructure and tunnels located in urban areas.

It is therefore necessary that these tunnels are designed to withstand a predictable and reasonable worst case scenario, taking into account their specific construction and engineering features. Since there is no universal approach to fire safety in tunnel design, the design team should exercise all due diligence and employ appropriate tools.

\section{Tunnel fire and evacuation model}

Fire dynamics simulator incorporating sub model Evac was selected for modelling of the tunnel fire and evacuation scenario. It is a CFD modelling software developed by the National Institute of Standards and Technology (NIST, USA) and VTT Technical Research Centre of Finland.

The tunnel in which the fire was modelled is Lucivna tunnel located on the D1 motorway near Strba, Slovak Republic.

\subsection{Fire dynamics simulator}

FDS is a computational fluid dynamics field computer model. FDS solves numerically a form of the Navier-Stokes equation appropriate for low-speed flow with an emphasis on smoke and heat transport from fires [5]. The second part of the package is Smokeview, a computer program for visualization of the results computed by FDS.

FDS uses rectangular geometry and divides the computational domain into a number of cells that compose a rectangular grid. All basic geometry is to fit within these cells; each cell represents uniform conditions. Besides the geometry one needs to set the fuel properties (chemical and physical), combustion reaction properties, ventilation and ambient conditions etc, known also as boundary conditions. FDS allows the user to work with logical devices that can undertake a specified action for a set condition.

This way, it is possible to model various detectors (smoke, heat, ionization), measure temperature, visibility obscuration and so on. Activation impulses from these devices then can trigger either some desired action (e.g. turn on ventilation) or activate sprinklers or start the discharge of a gaseous suppressant into the protected space. Results, point or planar, can be represented in numerical form or visually in Smokeview for planar results.

FDS version $6.0 \mathrm{RC} 3$ was used to model the case for this paper.

\subsection{FDS + Evac}

FDS+Evac is a combined agent-based egress calculation model and a Computational Fluid Dynamics (CFD) model of fire-driven fluid flow, where the fire and egress parts are interacting. FDS+Evac models the egress of the agents using continuous space and time, but the building geometry is fitted to the underlying rectilinear mesh. FDS+Evac uses simple rules and artificial intelligence to model the exit selection processes of the evacuees [6]. Evac is embedded in the FDS executable and is invoked automatically when an FDS input file contains Evac commands.

FDS+Evac version 2.5.0 included with FDS version 6.0 RC was used to model the case for this paper.

In FDS-Evac, there are two main parts of an evacuation scenario. Firstly, one needs to specify the geometry of the modelled case and assign specific boundary conditions. Secondly, the number and characteristics of the evacuees are required.

As part of the geometry of an evacuation case, a dedicated evacuation mesh is required. This is essentially a two-dimensional mesh as it has only one cell in the $\mathrm{z}$ direction. Specific exit boundary conditions have to be assigned to areas which are to be used as egress points (removal of agents from the calculation). Any part of the fire geometry that is to form an obstruction must extend itself through the evacuation mesh.

To define the response and behaviour of the evacuees (agents) a wide range of properties is available. This includes the dimensions of the agents, their response and speed, social force parameters etc.

\subsection{Modelled tunnel}

As mentioned above, the modelled tunnel is an existing road tunnel in the Slovak Republic. It was selected as its geometry is nearly rectangular and the length of the tube is $250 \mathrm{~m}$. Due to its length ventilation is natural via the portals. Each tube has a width of approx $12 \mathrm{~m}$ and a height of approximately $6 \mathrm{~m}$. The walls of the tunnel are constructed of reinforced concrete approximately $1 \mathrm{~m}$ thick. Only one of the two tunnel tubes was modelled as they are identical and completely separated. A fire in one of the tubes would therefore not affect the other tube, unless a structural failure occurred.

In order to achieve reasonable accuracy and computational times three, partially-overlapping, computational grids (meshes) were employed.

The main fire mesh had cells with dimensions $0.25 \mathrm{~m} \times 0.25 \mathrm{~m} \times 0.25 \mathrm{~m}$ and was used to model the central section of the tunnel, where the fire source was located. This section covered a length of $30 \mathrm{~m}, 15 \mathrm{~m}$ in each direction from the fire centreline.

A coarser mesh, with a cell of $0.5 \mathrm{~m} \times 0.5 \mathrm{~m} \times 0.5 \mathrm{~m}$, covers the rest of the tunnel tube on each end of the fire mesh. These meshes have a $2.5 \mathrm{~m}$ overlap with the fire mesh, to allow for uninterrupted flow.

This mesh setup resulted in a total of 267840 cells in the computational domain. 
The boundaries of the computational domain corresponded with the tunnel walls, floor and ceiling. To simulate the discharge of smoke from the tunnel portals the computational domain was extended by $3 \mathrm{~m}$ from each portal. This part of the computational domain was assigned an open boundary condition, meaning that smoke and air could flow freely from and into the tunnel.

Apart from lighting and signage, there is no further equipment installed in the tunnel. It represents a base modelling scenario, in which the fire is the primary driving force for all flows within the tunnel.

\subsection{Fire scenario}

A fully-laden van fire was simulated. The footprint of the fire was $10 \mathrm{~m}^{2}(5 \mathrm{~m} \times 2 \mathrm{~m})$ and its growth was simulated using the t-squared 'ultrafast' fire [7], which is the recommended design fire for tunnel scenarios [8]. The fire was set up in the following phases:

$\begin{array}{lll}\text { Growth phase } & 0-330 \mathrm{~s} & 020 \mathrm{MW} \\ \text { Fully-developed phase } & 330-2130 \mathrm{~s} & \text { 20MW constant } \\ \text { Decay phase } & 2130-2700 \mathrm{~s} & 200 \mathrm{MW}\end{array}$

The development of the fire and rate of burning is depicted in Fig. 1. After integration, the total mass of fuel burned during the simulation equals approximately to $1125 \mathrm{~kg}$ of fuel which is a very realistic assumption for a fully-laden van including its interior materials.

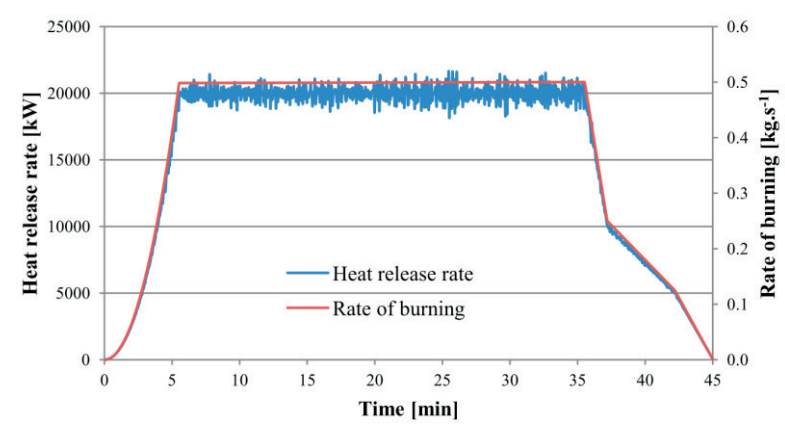

Fig. 1 Development of heat release and burning rates

The type of fuel was selected a mixture of plastics, namely polyethylene, polypropylene and polycarbonate. This way, it reflects the nature of the transported goods as well as interior materials.

Although of a significant size, the above fire represents a less severe tunnel fire, which according to the literature [9] can achieve 100MW and more.

\subsection{Evacuation scenario}

An evacuation scenario was specified in addition to the fire scenario described above. 60 evacuees (estimate based on the number of vehicles in the tunnel) were placed in one half of the tunnel length. One half of the tunnel length was assumed due to the fact that the vehicles after the fire (in the direction of travel) would continue to drive, leaving the second half of the tunnel length empty.

There were a total of 24 vehicles placed in the tunnel, representing obstructions for the evacuees. The initial distribution of the vehicles and evacuees (random) is shown in Fig. 2. The evacuation computational grid had 75000 cell $(1250 \times 60)$ and covered the same geometrical area (X-and Y-axis-wise) as the fire meshes.

The start of the evacuation was set to 180 s from the ignition of the fire. At this time the smoke travels approximately $3 / 4$ of the length from the fire to a portal, i.e. the presence of fire would be apparent. This was based on the outcomes of the model in [2]. Where smoke density exceeded prescribed levels, the evacuees started egress before the preset 180s start point.

\subsection{Measured quantities}

Measurements of various quantities were taken during the simulation. These measurements were taken at the fire centre point $(0 \mathrm{~m}), 5 \mathrm{~m}, 10 \mathrm{~m}, 20 \mathrm{~m}, 50 \mathrm{~m}$ and $100 \mathrm{~m}$ away. At each of these locations, there was a tree of thermocouples, located at $1 \mathrm{~m}, 2 \mathrm{~m}$, $3 \mathrm{~m}, 4 \mathrm{~m}, 5 \mathrm{~m}$ and $6 \mathrm{~m}$ above floor level.

Temperatures of the ceiling were recorded to establish the destructive potential of the fire. Other temperature measurements were taken at $2 \mathrm{~m}$ above floor level to assess tenability levels and potential danger to evacuating persons.

To further assess tenability and impact on the evacuees, the levels of carbon dioxide- $\mathrm{CO}$, and smoke movement were monitored; the first in the form of point measurements at $2 \mathrm{~m}$ above floor level, the latter visually through Smokeview.

Additional data were available from the evacuation calculations.

\section{Results}

As mentioned above, the first measured quantity was the temperature of the ceiling, the development of which is shown in Fig. 3.

The temperatures measured directly above the fire, increased sharply during the first five minutes, following the trend of the heat release rate. The concrete spalling temperature of around $300^{\circ} \mathrm{C}$ is therefore quickly exceeded; dangerous explosive 
deterioration of the concrete structure is expected to occur at this rate of temperature increase and the temperature achieved.

Another conclusion that may be drawn from the ceiling temperatures is the effect on steel pre-stressing or reinforcing members inside the concrete structure. The critical temperature of steel (around $400-450^{\circ} \mathrm{C}$ ) may be achieved, depending on the location - depth, which leads to a loss of strength and potentially structural integrity.

On the other hand, the temperature sharply decreases with the increasing distance from the fire. Even $5 \mathrm{~m}$ away from the fire the temperature drops below critical values (around 200$250^{\circ} \mathrm{C}$ ), meaning that any structural damage to the tunnel would be most likely of a local character. This is of course subject to the fire not spreading to further vehicles in its vicinity, in which case the damage would be of a greater extent.

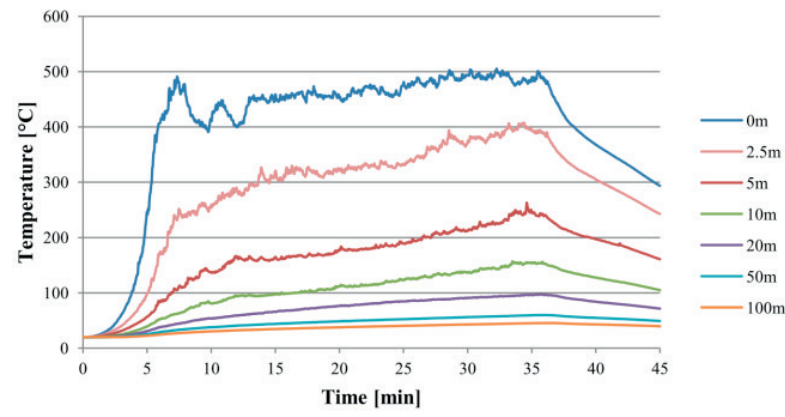

Fig. 3 Ceiling temperatures at various distances from fire

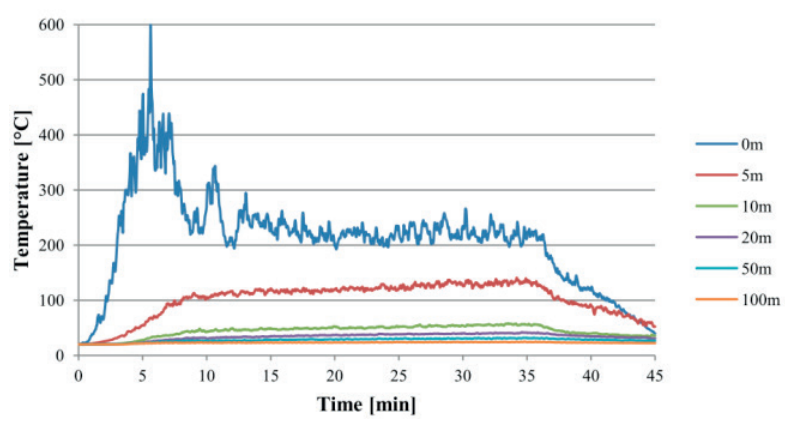

Fig. 4 Temperatures at $2 m$ above floor at various distances from fire

Temperatures at $2 \mathrm{~m}$ above floor level (Fig. 4) show, that except for the fire itself, tenability limits are exceeded only in the location $5 \mathrm{~m}$ away from fire. In this location, the maximum temperature is peaking at around $130^{\circ} \mathrm{C}$. Further away from the fire, the temperatures decrease to levels which are not life threatening $\left(50^{\circ} \mathrm{C}\right.$ and less $)$. This is caused by the current of fresh air, which is being drawn from the portals towards the fire.

To establish whether radiative fire spread would take place the intensity of radiative heat flux was monitored in the vicinity of the fire. The results of these measurements are shown in Fig. 5. The critical value of radiative heat flux (shown in dashed line), below which fire spread should not occur is $10 \mathrm{~kW} \cdot \mathrm{m}^{-2}[10]$.

It is clear from the above figure that there is a significant potential for fire spread via radiation to the vehicles in the vicinity of the fire. The critical value of $10 \mathrm{~kW} \cdot \mathrm{m}^{-2}$ is exceeded on the entire front face of the first vehicle located $3 \mathrm{~m}$ away from the fire; even higher intensities are recorded on the vehicle to the side of the fire. It is therefore likely that the fire would spread to these vehicles and grow beyond 20MW. This highlights the importance

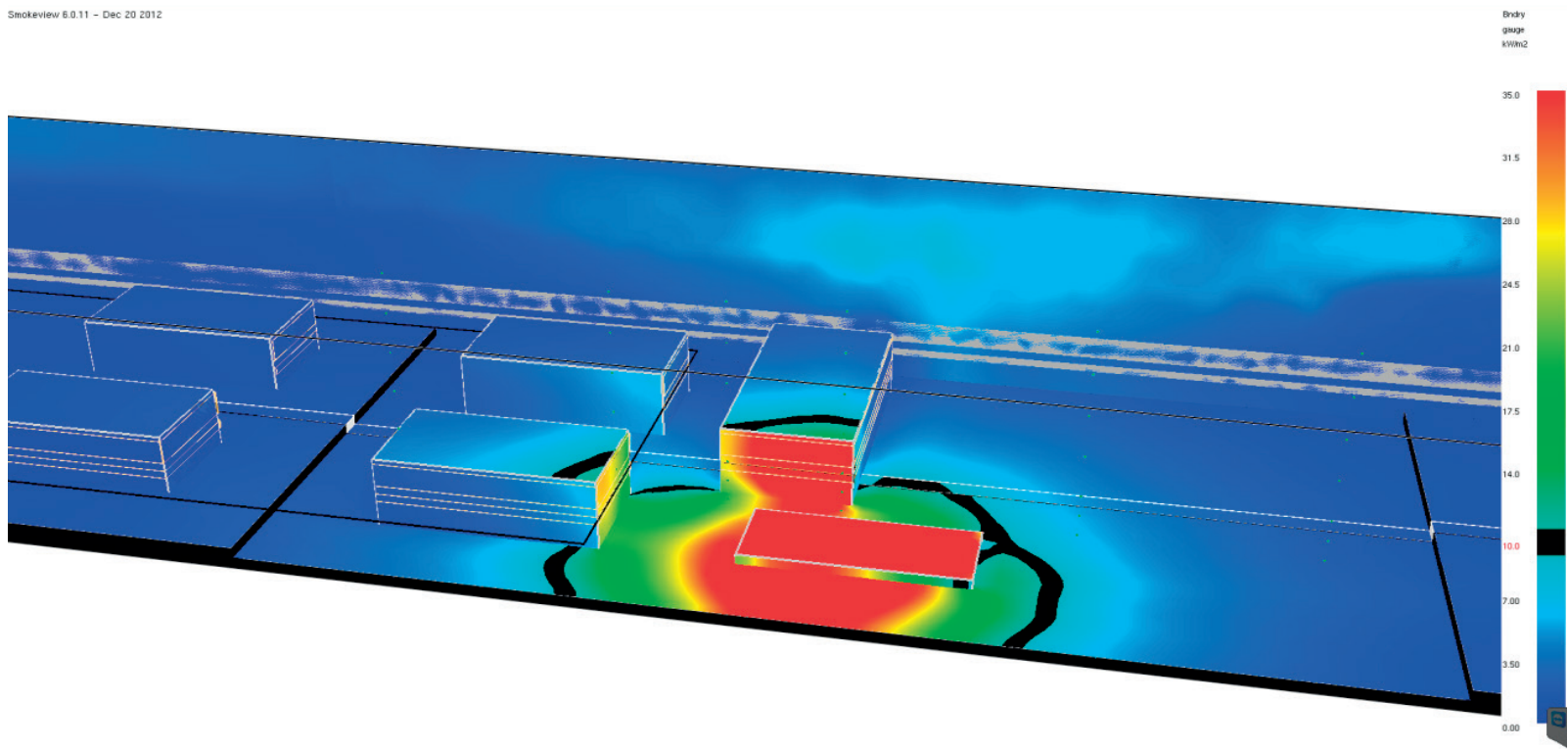




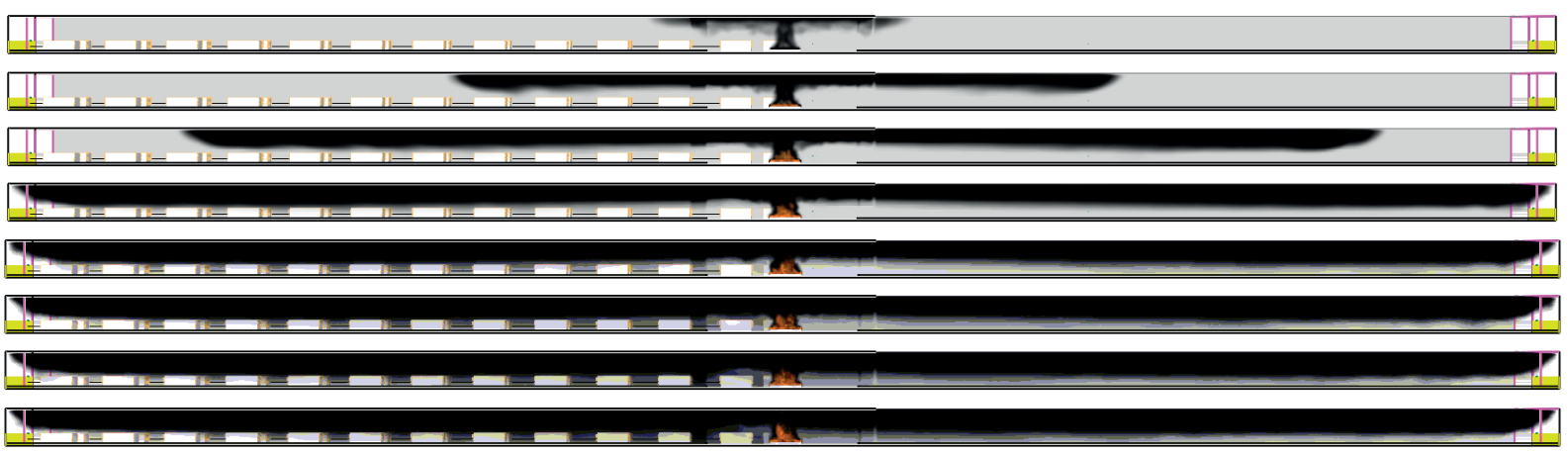

Fig. 6 Smoke movement inside the tunnel 60-480s (60s intervals)

of adequate fire scenario selection - isolated accident vs accident involving a number of vehicles.

With the exception of the fire area and its very close proximity $(2 \mathrm{~m})$, the $\mathrm{CO}$ levels monitored at $2 \mathrm{~m}$ above floor level did not exceed $11 \mathrm{ppm}$ during the simulation. Such levels pose no threat to the evacuees and there is no danger of their incapacitation. Again, this is caused by the fresh air current flowing towards the fire.

The visualisation of the simulation shows that there would be some smoke present in the lower layer of incoming air. This is caused by the turbulent mixing on the boundary between hot (smoke) and cold (fresh air) layers. In Fig. 6 a sequence of images illustrates how smoke is being drawn from the upper layer by the incoming air current. This is particularly obvious at the portals. The presence of smoke may of course negatively affect orientation and evacuation.

The evacuation simulation indicates that for this particular scenario (size of fire, geometry of tunnel, number of evacuees and their distribution) all the evacuees (also referred to as agents in FDS+Evac) were able to leave the tunnel safely.

Figure 7 shows the progress of the evacuation in time from the start of the fire. The evacuees commenced evacuation after 180s (see Section 2.5 for details) and the ones located near the tunnel entrance portal left almost immediately. Then the number of the evacuees decreased steadily with time indicating that the widths of the evacuation routes were sufficient. The evacuation time was therefore a function of the distribution and travel speed of the evacuees. Apart from two, all the evacuees had chosen to egress via the tunnel entrance portal, i.e. away from the fire, which is logical and expected behaviour.

To establish the level of evacuees' exposure to toxic combustion products FDS+Evac calculates so-called fractional effective dose (FED) which is a cumulative rate of all the toxic combustion products. When this rate reaches the value of 1 , the affected agent is 'dead', i.e. it has been exposed to a lethal dose of noxious substances. Fig. 8 shows that none of the evacuees experienced a lethal or near-lethal exposure. The FED increases with the duration of exposure and concentration of noxious gases which is directly dependent on the size of the fire. When the graph in Fig. 8 is compared with the smoke distribution in Fig. 6, the growing smoke layer corresponds with the increase in FED. The sharp decrease of the FED curve is caused by the fact that all the evacuees left the tunnel safely and are no longer included in the calculation.

The visualization of the evacuation (via Smokeview) is also available, however, its format is not adequate for paper presentation; it shows the movement of the individual agents.

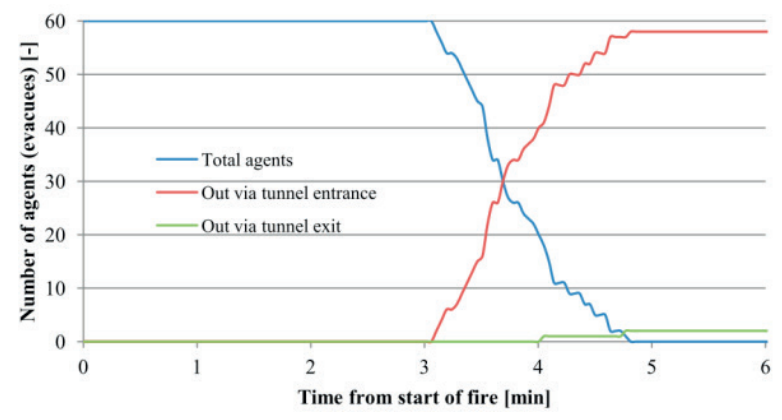

Fig. 7 Evacuation progress and usage of tunnel entrance and exit

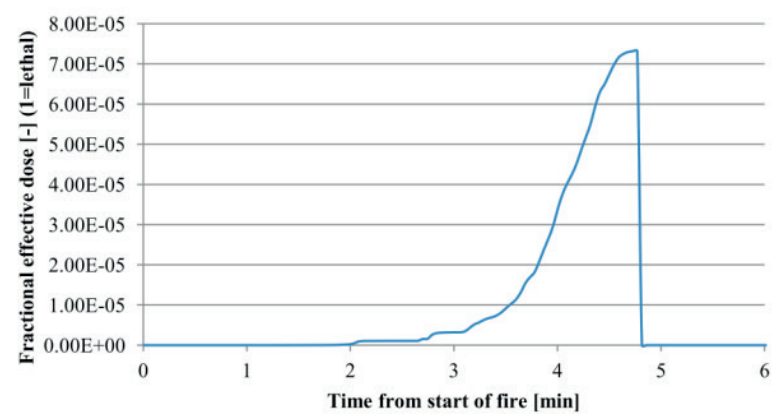

Fig. 8 Maximum fractional effective dose of toxic combustion products

\section{Conclusion}

Computer fire and modelling, namely Fire dynamics simulator + Evac, has been applied to a simple realistic scenario. The aim of the model was to demonstrate the capabilities of computer modelling in this area as well as evaluate the fire safety of an 
existing tunnel. The modelled fire was a fire of a van carrying plastic goods.

The results show that even a relatively small (on this scale) initial fire has the potential to grow significantly larger if other vehicles are sufficiently close. Radiative heat transfer plays a major role in this.

As regards structural integrity, the modelled fire would most likely cause some damage to the structure of the tunnel. The extent of the damage would be limited to the actual area of fire and its immediate vicinity.
The evacuation model results indicate that all persons inside the tunnel should be able to evacuate safely and reasonably fast. There should be no dangerous concentrations of combustion products, only some smoke in the lower layer, due to the turbulent nature of the air and smoke currents.

Overall, the fire scenario used as a basis for this paper should not pose a significant threat to the tunnel construction and persons located inside during the accident.

\section{References}

[1] MOZER, V.: Predicting Fire Severity in Tunnels, Proc. of conrefence Transcom 2013, Zilina, University of Zilina: Edis, 2013.

[2] MOZER, V., et al.: Fire Safety in Tunnels Forming Part of Critical Infrastructure, Proc. of conference ICCST 2013, Medellin, University of Antioquia, 2013. (to be published)

[3] RISTVEJ, J., ZAGORECKI, A.: Information Systems for Crisis Management - Current Applications and Future Directions, Communications: Scientific Letters of the University of Zilina, No. 2, vol. 13, 2011, p. 59-63, ISSN: 1335-4205.

[4] Transportation Research Board, Making Transportation Tunnels Aafe and Secure, NCHPRP Report 525, vol.12 Washington DC, Transportation Research Board, 2007.

[5] MC GRATTAN, K. et al.: Fire Dynamics Simulator (Version 6) - User's guide, Maryland, NIST, 2012.

[6] KORHONEN, T., HOSTIKKA, S.: Fire Dynamics Simulator with Evacuation: FDS+Evac Technical reference \& User's guide, Finland: VTT, 2010.

[7] DI NENO, P. J.: SFPE Handbook of Fire Protection Engineering, $3^{\text {rd }}$ ed. Maryland, SFPE, 2002

[8] BROOKS, R., et al.: Design of Smoke Ventilation Systems for Loading Bays \& Coach Parks - a Guide for System Designers, Reading, Smoke control association, 2010

[9] MAEVSKI, Y. I.: Design Fires in Road Tunnels, Washington DC, NCHRP, 2011

[10] NFPA 92B Standard for Smoke Management Systems in Malls, Atria, and Large Spaces, 2009 ed. Massachusetts, 2009. 